\title{
Die Schädigungen des Ohres durch Geschoßexplosion.
}

Von Priv.-Doz. Dr. Rudolf Hoffmann (München),

z. Z. Oberarzt a. K. bei der deutschen Militärmission in Konstantinopel.

Von der Häufigkeit der Detonationsschädigungen des Ohres in dem jetzigen Kriege werden wir kaum je ein richtiges Bild bekommen, da die Mehrzahl der davon Betroffenen eine gewisse Beeinträchtigung des Hörvermögens und etwas Ohrensausen als selbstverständlich hinnimmt und nur bei stärkeren Störungen sich zum Arzt meldet. Diese treten hauptsächlich ein, wenn eine Granate oder Mine in der Nähe einschlägt. Der vor oder in dem Graben oder im Unterstande sich aufhaltende Soldat wird dann meist geworfen, d. h. er fliegt gegen eine Böschung oder Wand, Sand stürzt nach, verschüttet ihn oft, er liegt vielleicht stundenlang auf dem Körper eines getöteten Kameraden, bis er ausgegraben wird. Wir haben also nicht nur die Symptome der Auris laesa zu erwarten, sondern auch mit der Shockwirkung und den Folgeerscheinungen einer Commotio cerebri zu rechnen. Der vorher noch gut hörende Soldat vernahm nach seinen Angaben das Pfeifen des kommenden Geschosses, sah wohl auch den Explosionsschein, hörte aber häufig keinen Knall. Das Bewußtsein war meist getrübt, aber selten ganz erloschen. Die Patienten geben an, daß sie eine Zeitlang ganz ,dösig" oder „,damisch" gewesen seien, das Gehör sei zuerst verloren gewesen, habe sich aber dann gebessert. Auffällig ist es, daß Störungen von seiten des $\mathrm{N}$. vestibularis außerordentlich selten sind. Als Schwindel wird sehr häufig die Verdunkelung des Gesichtsfeldes bezeichnet, richtige Gleichgewichtsstörungen sind sehr selten. Dagegen machen sich Ohrgeräusche höchst unangenehm bemerkbar und beunruhigen den Patienten oft mehr, als die Einbuße an Gehör oder die Blutung aus dem Ohr. Fehlt letztere, so macht ihn das Austreten von.Luft aus dem Ohr beim Schneuzen darauf aufmerksam, daß das Trommelfell einen Riß bekommen hat. Ausfluß von seröser Flüssigkeit aus dem Ohr sofort nach dem Unfall tritt nur dann auf, wenn zurzeit eine frische Mittelohrentzündung bestand, oder wenn bei einer Schädelfraktur Liquor durchs Ohr abfließt.

Befund. Sieht man einen Mann, der verschüttet war, am selben oder nächsten Tage, so fällt meist sein starrer, verstörter Gesichtsausdruck auf, oft wird man an das Wort vom ,,kristallisierten Schrecken", das beim Morbus Basedowii geprägt wurde, erinnert: in der Tat zeigt der Patient oft die Zeichen eines Basedowoids: weitgeöffnete Lidspalte, leichtes Hervortreten der Augäpfel, seltenen Lidschlag, Pulsbeschleunigung, Neigung zum Erschrecken und Schwitzen. Zuweilen bestand eine Druckempfindlichkeit der Schilddrüse (Kapselspannung?).

Trommelfellbild. Die Annahme, daß der Schädigung der Funktion die Veränderung am Trommelfell entspricht, wäre irrig. In mehr als der Hälfte der Fälle fand ich ein unverletztes Trommelfell. Stark geschädigt ist fast immer nur das der Explosionsstelle zugekehrte Trommelfell, das andere zeigt entweder normales Aussehen oder leichte Injektion und subepitheliale Blutungen. Im Gehörgang lag nicht selten Kreide in Bröckeln oder so fein pulverisiert, daß man hätte glauben können, es wäre Borpulver eingeblasen worden. Der knöcherne Abschnitt des Gehörganges weist oft Blutgerinnsel auf, und Blutungsreste decken auch meist die Umgebung der Perforation. Das übrige Trommelfell kann alle Uebergänge vom normalen Aussehen bis zur stärksten Rötung zeigen.

Sitz der Perforation. In weitaus der Mehrzahl derFälle sitzt die Perforation an der Stelle des dreieckigen Reflexes, d. h. 
dort, wo am normalen Trommelfell eine senkrecht zum einfallenden Lichte gelegene Ebene die Wellen ins Auge reflektiert. Zuweilen ist es nur ein gerader oder gelappter Riß, häufig aber greifen die Grenzen"des Loches weiter, nehmen die untere Hälfte des Trommelfells oder noch größere Bezirke der Membran ein. Am seltensten sitzt die Perforation vorn oben. Trifft man eine isolierte Perforation hinten oben oder in der Membrana Shrapnelli, so geht man wohl in der Annahme nicht fehl, daß vor dem Unfall schon eine durch Tubenprozesse usw. bedingte Spannungsanomalie oder eine Narbe an der betreffenden Stelle bestand. Oft zeigt die Untersuchung des unverletzten Ohres parallele Reflexverschiebungen, die unsere Vermutung bestätigen.

Die For m der Perforation ist meist oval, zuweilen schlitzförmig; knopflochartige sah ich einige Male im vorderen oberen Quadranten dicht am Hammergriff entlanglaufen. Die großen Defekte zeigen nieren- und herzförmige Gestalt oder betreffen das ganze Trommelfell. Auffallend häufig sehen die Löcher wie ausgestanzt aus; zerfetzte, zackige Ränder findet man viel seltener, als man sie nach den Bildern der traumatischen Trommelruptur, wie man sie von Friedenszeiten her kannte, erwarten konnte. Die gelbgraue Promontorialwand kontrastiert gegen den roten Perforationsrand. Sie ist und bleibt in der Mehrzahl der Fälle reizlos. Zuweilen sind einige senkrecht verlaufende Gefäßreiserchen injiziert.

Bei einem durch Sprengung geschleuderten Soldaten sah ich neben Blutabfluß aus dem Ohre das Trommelfell durch bläulich durchschimmerndes Blut wurstartig rings um den Umbo sich vorwölben: ein Hämatotympanum bei Basisfraktur. Bei einem anderen sah ich den Liquor durch eine Perforation der Membrana Shrapnelli abtropfen.

Die Beeinträchtigung des Hörvermögens schwankt in weiten Grenzen. Taubheit für Sprache ist selten, in einem Falle trat nach mehreren Tagen beiderseits Ertaubung unter den Erscheinungen einer Labyrinthblutung auf. In typischer Weise wird die Zahl ,55" am schlechtesten gehört. Oft sind die Ohrengeräusche so stark, daß sie das Ergebnis der Hörprüfung wesentlich beeinflussen, ihre Klangfarbe wird als Zischen, Pfeifen, Singen, Klingen, seltener als Sausen, Brausen oder Donnern bezeichnet. Der Ton der auf den Scheitel gesetzten Stimmgabel wird ins gesunde Ohr lateralisiert, der Rinnesche Versuch fällt positiv aus. Die Knochenleitung ist verkürzt und die obere Hörgrenze minder oder mehr eingeengt, zuweilen liegt die Grenze nicht mehr in der Galton-Pfeife.

Spontaner Nystagmu.s bestand sehr selten, die Prüfung des Ganges mit verschlossenen Augen und der Zeigeversuch gaben keine einheitlichen Resultate. Die Untersuchung des kalorischen Nystagmus verbot sich aus naheliegenden Gründen.

Diagnose. Ist das Trommelfell unverletzt und sind die Angaben des Patienten nicht ganz zuverlässig, so ist die Differentialdiagnose gegen eine schon vorher vorhandene Berufsschwerhörigkeit durch den funktionellen Befund nicht $\mathrm{zu}$ erbringen, er ist bei ihr derselbe wie bei der Detonationslabyrinthose. Und doch ist es aus praktischen Gründen bezüglich späteren Entschädigungsansprüchen wichtig, das Kriegstrauma sicherstellen zu können. Ich habe an anderer Stelle ${ }^{1}$ darauf hingewiesen, daß wir bei Detonationslabyrinthosen stets Sensibilitätsstörungen am Ohre finden. Auf Befragen gaben die Patienten an, die Ohrmuschel sei pelzig, wie eingeschlafen oder wie tot. Prüft man die Sensibilität des äußeren Ohres, so findet man das Gefühl für spitz und stumpf, kalt und warm, für Berïhrung der Härchen der Ohrmuschel und der Haut herabgesetzt. Bei starken Detonationslabyrinthosen verursacht der tiefe Nadelstich ins Ohrläppchen keinen Schmerz, während der Simulant unangenehm berührt zusammenfährt, wenn die Prüfung ohne vorherige Fragestellung ausgeführt wird. Der hypästhetische Bezirk beschränkt sich aber nicht nur auf das Ohr, sondern erstreckt sich oft auf die Umgebung des Ohres, auf die Wange, die Stirn. Es ist darauf zu achten, ob etwa Sensibilitätsstörungen einer ganzen Körperhälfte vorliegen. Die Intensität der Gefühlsherabsetzung entsprach stets der Größe des Ausfalls an der oberen Tonskala. Mit der Minderung desselben besserte sich auch das Gefühl an der Ohrmuschel, aber nach Jahresfrist waren Gefühlsstörungen nachweisbar.

1) M. m. W. 1915 .
Bei Soldaten, die bisher Detonationen nicht ausgesetzt waren, sowie bei Eisenbahnbeamten mit Berufsschwerhörigkeit fand ich das Symptom nicht. Bei sogenannten Otosklerosen fehlt der Kitzelreflex, der Gehörgang, die Gefühlsqualitäten an der Ohrmuschel sind nicht beeinflußt.

In Zusammenhang mit diesem Symptom dürfte ein weiteres stehen, das ich bei der Detonationslabyrinthose fand: ein Ausfall in der Orientierungsfähigkeit bezüglich der Schallrichtung. Soweit meine Erfahrung reicht - ich konnte im Felde keine diesbezüglichen Untersuchungen anstellen -, besteht ein solcher bei anderen einseitigen Ohrerkrankungen nicht in derselben Weise. Dann ist wohl der Rückschluß erlaubt, daß für die Fähigkeit, die Schallrichtung zu bestimmen, die Sensibilität des äußeren Ohres von besonderer Bedeutung ist. Dafür spricht die Versorgung des äußeren Ohres mit Hautnerven aus den Stämmen des $\mathrm{N}$. vagus, $\mathrm{N}$. trigeminus, $\mathrm{N}$. auricularis magnus und $N$. occipitalis minor. Die von den Wellen senkrecht getroffenen Hautstellen des äußeren Ohres werden die stärkste sensible Erregung erhalten, dieses Feld können wir durch Kopfwenden vergrößern. Gewiß spielt auch das Gelenk- und Muskelgefühl der Schalleitungskette im Mittelohr eine Rolle, deren Spannungszustand wohl sicher durch die sensiblen Hautnerven des äußeren Ohres orientiert wird.

Daß Störungen im Bereich der Binnenmuskeln oder der Gelenke vorliegen, dafür spricht ein weiteres von mir gefundenes Symptom, das ich bei anderen Mittelohraffektionen nicht beobachtete. Bei vielen Patienten, die über Schmerzen im $\mathrm{Ohr}$ beim Schlucken und besonders beim Aufstoßen klagten, trat dieser Schmerz bei festem Schluß der Augenlider auf. Es handelt sich um eine Mitbewegung des auch vom $\mathrm{N}$. facialis versorgten $M$. stapedius, dessen Aktion sich meist auch durch dumpfes Flattern im $\mathrm{Ohr}$ verrät. Genauere Untersuchung dieser Erscheinung, besonders bezüglich der Veränderung des Tones der vor die Nase gehaltenen (Poli ízer) und der auf den Scheitel gesetzten (Ludw. Hoff mann) tönenden a-Stimmgabel beim Schluckakt, stehen mir leider nicht in genügender Zahl zu Gebote.

Ein Hinweis darauf sei erlaubt, daß der beim Bestreichen symmetrischer Hautstellen am Tragus entstehende Ton am detonationsverletzten $\mathrm{Ohr}$ höher ist als am gesunden bzw. besseren Ohre. Musikalische Patienten gaben an, daß die Klangfarbe der Töne des Instrumentes, z. B. der Orgel, verändert, unrein erscheinen.

Verlauf der Erkrankung. Sekretion aus dem Ohre tritt entweder garnicht oder erst nach Ablauf von 24 Stunden auf. Zeigt sich bald nach dem Unfall seröser Ausfluß, so hat, wie schon gesagt, das Trauma ein bereits akut entzündetes $\mathrm{Ohr}$ betroffen, oder es handelt sich um Liquorabfluß infolge Basisfraktur. In der Mehrzahl der Fälle versiegt der Ausfluß bald; hat jedoch eine Sekundärinfektion stattgefunden, so wird er meist bald sehr reichlich und schleimig-eitrig. Die Erfahrung lehrt, daß die entzündlichen Mittelohrprozesse im Anschluß an Detonationsverletzungen sehr stark zu Komplikationen neigen. Diese Patienten erhielten stets Urotropin, bei Pneumokokkeninfektion wäre Optochin zu versuchen.

Sie bedürfen aufmerksamer Beobachtung.

Bleibt eine Infektion fern, so bessert sich bald das Gehör; die Stiche im Ohr beim Schlucken bleiben noch einige Zeit, am längsten beunruhigten den Patienten die Ohrgeräusche. Die Perforation zeigt in verschiedenem Maße, das von Art der Zerreißung, Konstitution des Trägers usw. abhängt, Neigung zum Verschluß. Der Rand bleibt getrübt und injiziert, während eine zarte Narbe allmählich das Loch verschließt. Frühestens nach vier Wochen sah ich die Perforation geheilt. Die Schädigung elastischer Elemente im Trommelfell manifestiert sich im Auftreten von Verkalkungen. Perforationen geringen Umfanges heilen oft spurlos aus.

Diagnose. Bei verletztem Trommelfell ist der Befund leicht richtig zu beurteilen, bei intakter Membran ist die Sensibilitätsstörung am äußeren Ohr das einzige Kriterium, welches uns die Abgrenzung der Detonationsverletzung gegen die Berufsschwerhörigkeit ermöglicht.

Wieweit es sich um funktionelle Störungen dabei handeln dürfte, ist eine offene Frage. Vielleicht ist die anfängliche totale Taubheit eine solche, wie die sie häufig begleitende Aphonie, Verdrängte Sexualaffekte nach Freud wird wohl niemand an- 
nehmen. Gegen den funktionellen Charakter spricht die allmählich eintretende Besserung, vor allem aber die parallel laufende Einschränkung an der oberen Tonskala, sowie die Einseitigkeit der Erkrankung. (Daß wir den Begriff des „Funktionellen" werden revidieren müssen, darauf deutet die Tatsache hin, daß ich unter etwa 50 sogenannten funktionellen Schließerparesen, die im Schützengraben entstanden waren, keine ohne organische Läsion der Stimmlippen sah.)

Die Beobachtungszeit ist zu kurz, um sagen zu können, wie groß ungefähr die Zahl der Detonationslabyrinthosen ist, welche restlos ausheilt. Sie wird um so größer sein, je rationeller die Behandlung des verletzten Ohres ist.

Behandlung. Das Unterlassen jeglichen therapeutischen Eingriffes gewährleistet einen unkomplizierten Heilverlauf. Ausspritzen des Ohres ist hier ein Kunstfehler, der dem Patienten unter Umständen das Leben kosten kann. Nichts ist geboten, als der Verschluß des Gehörganges mit einem sterilen Wattepfropf. Die Streifcheneinlage ist zu verwerfen, weil die Stofffasern, durch die Perforation reichend, bis ins Mittelohr zu liegen kommen können. Der.Patient ist zur Vorsicht beim Waschen zu ermahnen, damit nicht Wasser ins Ohr läuft, die Benutzung von Brausebädern ist zu verbieten. Er erhält drei Tage Bettruhe verordnet, um die fast stets bei frischen Fällen vorhandene Shockwirkung abklingen zu lassen. Tinctura Valerianae wurde jedesmal gegeben, nicht, als ob ich ihr eine besondere Heilkraft zugutehielte, aber der Soldat empfindet die eindringliche Vorschrift, dreimal täglich genau 25 Tropfen zu nehmen, und den Hinweis auf ihre vorzügliche Wirkung als eine Fürsorge, die ihm in seinem Zustande besonders wohltut. Auch häufig vorgenommene Hörprüfungen, die ihm eine, wenn auch langsam fortschreitende Besserung des Hörvermögens zeigen, wirken suggestiv günstig, besonders auch auf das Ohrensausen, das oft zum Teil der Ausdruck seiner Hörunsicherheit ist. (Ist das Sausen eine Erscheinung einer allgemeinen Gefäßneurose, so schwindet bei tiefster, dann angehaltener Inspiration der Radialpuls sogleich oder nach wenigen Sekunden.)

Der Kranke ist vor dem Zusammensein mit infektiösen Kranken zu schützen, eine Tonsillitis kann ihn aufs schwerste gefährden. Bestehende Katarrhe der oberen Luftwege sind entsprechend zu behandeln. Das Wärterpersonal ist anzuweisen, laut und deutlich zu sprechen, um den Patienten das Unangenehme seiner Gehörbeschränkung nicht empfinden zu lassen.

Stark Schwerhörige und Ertaubte sollen baldigst versuchen, das Ablesen vom Munde mit Hilfe eines Spiegels zu erlernen.

Der beste Schutz für beschädigte Ohren ist, den Detonationen, d. h. dem Grabendienst fernzubleiben. Das läßt sich leider nicht durchführen; ist die Hörweite auf dem einen $\mathrm{Ohr}$ mehr als $400 \mathrm{~cm}$ (für die Zahl 55 in Flüstersprache), auf dem geschädigten mehr als $100 \mathrm{~cm}$, dann muß der Mann wieder hinaus. Wenn die Hörweite geringer ist, würde er stark gefährdet sein, weil er den Abschuß feindlicher Gewehrgranaten nicht hören und deshalb nicht rechtzeitig in Deckung gehen kann.

Als Ohrschutz gab ich ein Antiphon, welches leicht herzustellen ist: Ein Wattebausch wird mit einem nicht zu dünnen Bindfaden umknotet. Das eine Ende des letzteren wird am Knoten kurz abgeschnitten, um das andere die Watte zur Form einer Olive gewickelt, in flüssiges Wachs getaucht und der Gehörgangsweite entsprechend modelliert. Dieses Antiphon sitzt reizlos im Ohr und setzt das Hörvermögen auf etwa $20 \mathrm{~cm}$ Flüstersprache für die Zahl 88 herab. Es erfüllt die Hauptbedingung: es ist schnell zu entfernen, auch die abgearbeitete Hand des Soldaten findet sogleich die an der (oder um die) Ohrmuschel hängende Schnur. Denn der draußen stehende Soldat ist unsicher, wenn er nicht jederzeit sofort im Bedarfsfalle in der Lage ist, sich in den Besitz seiner optimalen Hörfähigkeit $\mathrm{zu}$ setzen.

Am Geschütz scheinen Kanoniere, die in einer bestimmten Stellung zur Rohrmündung stehen, besonders gefährdet. Sie sollten dahin belehrt werden, daß sie nicht nur den Mund zu öffnen, sondern so weit als möglich aufzumachen haben. Denn nur dann ist ein Schluckakt und damit ein Oeffnen der Tube unmöglich, sodaß die im Mittelohr eingeschlossene Luft wie ein elastisches Luftkissen federnd dem von außen ans Trommelfell tretenden Luftdruck entgegenwirken kann. 